\title{
Mitofusin-2 prevents skeletal muscle wasting in cancer cachexia
}

\author{
QIU-LEI XI $^{1 *}$, BO ZHANG ${ }^{1 *}$, YI JIANG ${ }^{1 *}$, HAI-SHENG ZHANG ${ }^{2}$, QING-YANG MENG $^{1}$, YING CHEN $^{2}$, \\ YU-SONG HAN $^{1}$, QIU-LIN ZHUANG ${ }^{1}$, JUN HAN ${ }^{1}$, HAI-YU WANG ${ }^{1}$, JING FANG ${ }^{2}$ and GUO-HAO WU ${ }^{1}$ \\ ${ }^{1}$ Department of Surgery, Zhongshan Hospital, Shanghai Medical College of Fudan University, Shanghai 200032; \\ ${ }^{2}$ Key Laboratory of Food Safety Research, The Institute for Nutritional Sciences, Shanghai Institutes for Biological Sciences, \\ Chinese Academy of Sciences, Shanghai 200031, P.R. China
}

Received April 16, 2015; Accepted July 28, 2016

DOI: $10.3892 / 01.2016 .5191$

\begin{abstract}
Cancer cachexia remains a leading cause of morbidity and mortality worldwide, despite extensive research and clinical trials. The prominent clinical feature of cancer cachexia is the continuous loss of skeletal muscle that cannot be fully reversed by conventional nutritional support, and that leads to progressive functional impairment. The mechanism underlying muscle loss in patients with cachexia is poorly understood. The present study analyzed 21 cancer patients with or without cachexia, and demonstrated that mitofusin-2 (Mfn2) was downregulated in the rectus abdominis of patients with cachexia, which was associated with body weight loss. In vitro cell experiments indicated that loss of Mfn2 was associated with atrophy of the $\mathrm{C} 2 \mathrm{C} 12$ mouse myoblast cell line. Furthermore, in vivo animal experiments demonstrated that cachexia decreased gastrocnemius muscle mass and $\mathrm{Mfn} 2$ expression, and overexpression of $\mathrm{Mfn} 2$ in gastrocnemius muscle was able to partially attenuate cachexia-induced gastrocnemius muscle loss. The results of the present study suggested that Mfn2 is involved in cachexia-induced muscle loss and may serve as a potential target for therapy of cachexia.
\end{abstract}

\section{Introduction}

Cachexia, which is the loss of body mass that cannot be reversed by nutrition, is frequently observed in patients with cancer. Cancer cachexia is a leading cause of morbidity and

Correspondence to: Dr Guo-Hao Wu, Department of Surgery, Zhongshan Hospital, Shanghai Medical College of Fudan University, 138 Yixueyuan Road, Shanghai 200032, P.R. China

E-mail: doctorwugh@163.com

Dr Jing Fang, Key Laboratory of Food Safety Research, The Institute for Nutritional Sciences, Shanghai Institutes for Biological Sciences, Chinese Academy of Sciences, 320 Yueyang Road, Shanghai 200031, P.R. China

E-mail: jfang@sibs.ac.cn

${ }^{*}$ Contributed equally

Key words: mitofusin-2, cancer, cachexia, muscle wasting mortality worldwide (1). The prominent clinical feature of cancer cachexia is the continuous loss of skeletal muscle that cannot be fully reversed by conventional nutritional support, leading to progressive functional impairment $(2,3)$. The skeletal muscle loss is associated with a reduced quality of life, as well as poor survival (4). The pathophysiology of cancer cachexia is characterized by a negative protein and energy balance (5); however, the underlying mechanism remains largely unknown. Previous studies have demonstrated that numerous signaling molecules and transcription factors are involved in the regulation of skeletal muscle mass $(6,7)$.

Mitochondrial function is crucial for the maintenance of the skeletal muscle (8). It has been demonstrated that suppression of mitochondrial function is sufficient to cause muscle wasting in adult animals (8). Mitochondrial function requires the coordination of mitochondrial fusion and fission processes that are referred to as mitochondrial dynamics $(9,10)$. A system of pro-fusion and pro-fission proteins regulates mitochondrial morphology and subcellular localization $(9,10)$. The fusion proteins mitofusin-1 (Mfn1) and mitofusin-2 (Mfn2) promote mitochondrial elongation and activity (11). As a major determinant of the fusion process, Mfn2 is a large GTPase that is integral to the mitochondrial outer membrane (12). It is essential for mitochondrial fusion during embryonic development and neuronal differentiation (13). In humans, mutations in the Mfn2 locus have been associated with numerous diseases, including Charcot-Marie-Tooth type 2A neuropathy, diabetes and Alzheimer's disease (14). Mfn2 is robustly expressed in muscle cells and its insufficiency has been associated with the fragmentation of the mitochondrial network, which is essential for the normal mitochondrial function (14). Inhibition of mitochondrial fission has been shown to inhibit muscle loss during fasting $(11,15)$. In addition, downregulation of Mfn2 has been observed in the muscle of obese and non-obese type 2 diabetic subjects $(16,17)$, and decreased mRNA expression levels of Mfn2 were observed in the skeletal muscle of tumor-bearing mice with severe cachexia (11). These results suggested that Mfn2 is involved in muscle wasting associated with diseases such as cancer cachexia.

The role of Mfn2 in skeletal muscle loss in cancer cachexia remains largely unknown. Therefore, the present study aimed to investigate the association between Mfn2 expression in skeletal muscle and body weight loss in patients with cancer cachexia. In addition, the potential role of Mfn2 in skeletal 
muscle atrophy and mass loss was evaluated by in vitro methods using the $\mathrm{C} 2 \mathrm{C} 12$ mouse myoblast cell line and in vivo animal experiments.

\section{Materials and methods}

Reagents. Monoclonal mouse Mfn2 antibody (cat. no. sc-100560; 1:100) was purchased from Santa Cruz Biotechnology, Inc. (Dallas, TX, USA). Polyclonal rabbit cytochrome $c$ oxidase subunit IV (COX IV; cat. no. AC610; 1:1,000) and monoclonal mouse cytochrome $c$ (Cyto C; cat. no. AC909; 1:200) antibodies were obtained from Beyotime Institute of Biotechnology (Haimen, China). Monoclonal mouse $\beta$-actin antibody (cat. no. A1978; 1:200) and tumor necrosis factor- $\alpha$ (TNF- $\alpha$ ) were purchased from Sigma-Aldrich (Merck Millipore, Darmstadt, Germany). Polyclonal goat anti-rabbit (cat. no. 35552; 1:5,000) anti-mouse (cat. no. 35502; 1:5,000) IgG secondary antibodies were purchased from Thermo Fisher Scientific, Inc., (Waltham, MA, USA). All other chemical reagents were standard commercial products of analytical-reagent grade.

Human skeletal muscle samples of cancer cachexia. Rectus abdominis muscle tissues were obtained from 21 patients undergoing primary surgical resection of gastrointestinal cancer at Zhongshan Hospital of Fudan University School of Medicine (Shanghai, China) between March and September 2012. The inclusion criteria were as follows: i) Patients had histologically documented cancers; ii) patients had not received prior anticancer treatment; and iii) patients had exhibited weight loss in the prior 6 months. Muscle tissue samples $(5 \times 5 \times 5 \mathrm{~mm})$ were fresh-frozen in liquid nitrogen immediately following resection, and were stored at $-80^{\circ} \mathrm{C}$. The present study was approved by the Ethics Committee of Zhongshan Hospital of Fudan University. Written informed consent approving tissue donation for research purposes was obtained from all patients prior to tissue collection.

Lentivirus. The vector plasmid for Mfn2 overexpression and control vector plasmid were prepared by GeneChem, Co., Ltd. (Shanghai, China). The lentivirus was produced by transient transfection of 293T cells with the vector plasmids, gag-pol packaging plasmid (Shanghai Institutes for Biological Sciences, Chinese Academy of Sciences, Shanghai, China) and envelope plasmid (Shanghai Institutes for Biological Sciences, Chinese Academy of Sciences) using Lipofectamine ${ }^{\circledR} 2000$ (Invitrogen; Thermo Fisher Scientific, Inc.), according to the manufacturer's protocol.

Cell culture. The $\mathrm{C} 2 \mathrm{C} 12$ mouse myoblast and HCT116 human colon cancer cell lines (Cell Bank of Shanghai Institutes for Biological Sciences, Chinese Academy of Sciences) were maintained in Dulbecco's modified Eagle's medium (DMEM; Thermo Fisher Scientific, Inc.) supplemented with $10 \%$ fetal bovine serum (Thermo Fisher Scientific, Inc.), $2 \mathrm{mM}$ L-glutamine, $100 \mathrm{U} / \mathrm{ml}$ penicillin and $100 \mu \mathrm{g} / \mathrm{ml}$ streptomycin. For the differentiation of $\mathrm{C} 2 \mathrm{C} 12$ cells, the cells $\left(2.5 \times 10^{5}\right)$ were grown in DMEM supplemented with $2 \%(\mathrm{v} / \mathrm{v})$ horse serum (Thermo Fisher Scientific, Inc.), 2 mM L-glutamine, $100 \mathrm{U} / \mathrm{ml}$ penicillin and $100 \mu \mathrm{g} / \mathrm{ml}$ streptomycin. Cells were then incubated with
TNF- $\alpha(0,2.5$ and $5 \mathrm{ng} / \mathrm{ml})$ and cultured at $37^{\circ} \mathrm{C}$ in an incubator with $5 \% \mathrm{CO}_{2}$ for 7 days.

Animals. Four-week-old male nude mice (BALB/cA-nu/nu) $(\mathrm{n}=22$; weight, $14.96 \pm 1.39 \mathrm{~g})$ were obtained from the Shanghai Laboratory Animal Center (Shanghai Institutes for Biological Sciences, Chinese Academy of Sciences) and maintained under pathogen-free conditions. The mice were housed in laboratory cages at $23^{\circ} \mathrm{C}$ and exposed to $12 \mathrm{~h}$ light/dark cycles with free access to food and water. All protocols invovling animals were approved by the Institutional Animal Care and Use Committee at the Institute for Nutritional Sciences (Shanghai, China). The mice were separated into four groups (5-6 mice/group), as follows: i) Tumor-bearing and blank lentivirus-treated mice (group 1); ii) tumor-bearing and $\mathrm{Mfn} 2$ overexpression lentivirus-treated mice (group 2); iii) tumor-free and blank lentivirus-treated mice (group 3); and iv) tumor-free and Mfn2 overexpression lentivirus-treated mice (group 4). The tumor-bearing mice were subcutaneously injected with $5 \times 10^{6}$ HCT116 cells (500 $\mu$ l) at each flank, whereas the tumor-free mice were injected with $500 \mu 1$ phosphate-buffered saline (PBS) as a control. Body weights, food intake, and tumor formation were assessed every 3 days. The mice were categorized as having severe cachexia when the body weights were $>2$ standard deviations (SDs) away from the means of the age-matched control mice. The mice with cachexia were treated with Mfn2 overexpressing lentivirus $\left(10^{8}\right.$ in $100 \mu$ PBS), which was injected intramuscularly into the gastrocnemius muscles every 3 days, four times in total. All mice were maintained for 40 days prior to euthanasia with $\mathrm{CO}_{2}$. Immediately following sacrifice, the gastrocnemius muscles were dissected and weighed, and then fresh-frozen in liquid nitrogen or fixed in paraformaldehyde. All mice were maintained and used in accordance with the guidelines of the Institutional Animal Care and Use Committee of the Institute of Nutritional Sciences.

Western blot analysis. Western blot analysis was performed as described previously (18). Briefly, frozen muscle tissue samples or cells were homogenized in Mueller buffer and the protein concentration was determined by the Bradford method. Crude tissue homogenate $(40 \mu \mathrm{g})$ was fractionated on $8-13 \%$ SDS-polyacrylamide gels, and the proteins were transferred onto polyvinylidene fluoride membranes overnight. The membranes were stained with Ponceau solution to verify that each gel had been equally loaded. Subsequently, the membranes were blocked overnight with 5\% milk in PBS containing 0.1\% Tween-20 (PBS-T), followed by incubation for $1 \mathrm{~h}$ at room temperature with anti-Mfn2 (86 kDa; 1:100), anti-COX IV (17 kDa; 1:1,000), anti-Cyto C (15 kDa; $1: 200)$ and anti- $\beta$-actin (43 kDa; $1: 200)$ primary antibodies. The membranes were then incubated for $1 \mathrm{~h}$ at $25^{\circ} \mathrm{C}$ with horseradish peroxidase-conjugated anti-rabbit and anti-mouse IgG (diluted 1:5,000 in 5\% milk in PBS-T), followed by detection of the bound secondary antibodies with enhanced chemiluminescence (GE Healthcare Life Sciences, Piscataway, NJ, USA). Images were digitally scanned, and blots were quantified by densitometry using ImageJ 1.46r software (National Institutes of Health, Bethesda, MA, USA).

Reverse transcription-quantitative polymerase chain reaction $(R T-q P C R)$. Isolation of RNA from the rectus abdominis muscle 
A

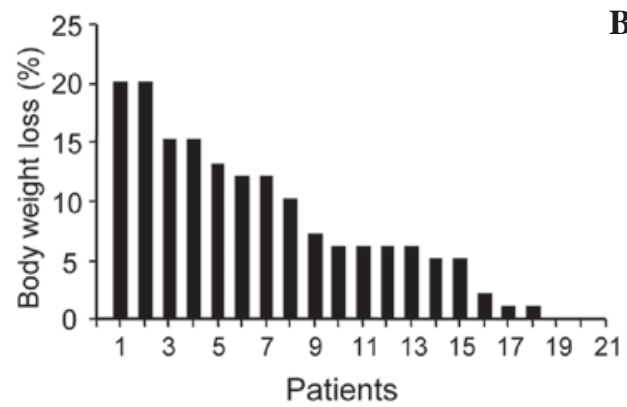

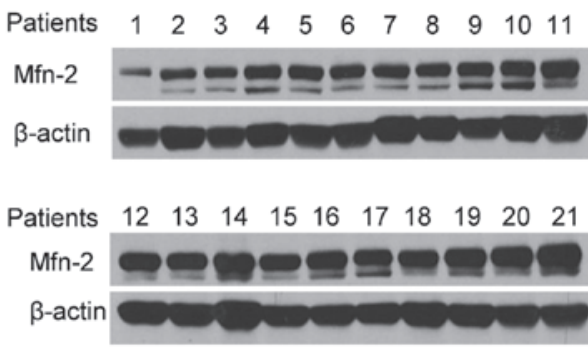

C

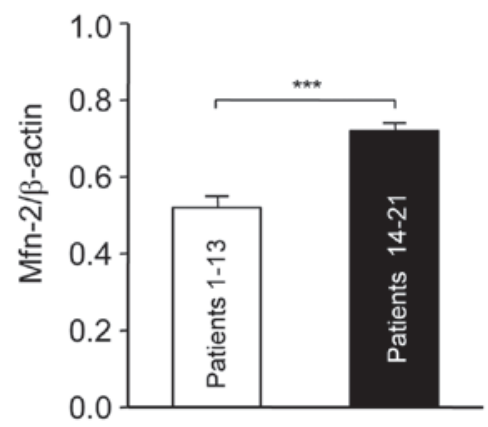

D

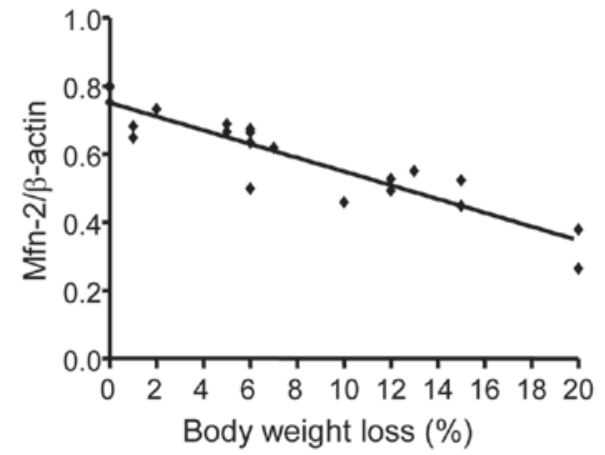

Figure 1. Downregulation of Mfn2 in the rectus abdominis of 21 patients with gastrointestinal cancer was associated with body weight loss. (A) Percentage body weight loss of the 21 patients in the 6 months preceding surgery. (B) The protein expression of Mfn 2 in the gastrocnemius of patients was assessed using western blotting. (C) The mRNA expression levels of Mfn2 were decreased in cancer patients with cachexia compared with those without cachexia, as determined using reverse transcription-quantitative polymerase chain reaction. The criterion for cachexia was $>5 \%$ weight loss. (D) Association between weight loss and the expression level of Mfn2 in the rectus abdominis of the 21 patients with gastrointestinal cancer was analyzed using GraphPad 5.0 software. Slope $=-0.02017$ (95\% confidence interval $=-0.02439$ to -0.01595$)$. Data are presented as the mean \pm standard error of the mean. ${ }^{* * *} \mathrm{P}<0.001$ vs. Patients $1-13$. Mfn2, mitofusin-2.

tissues was performed using the EZ-10 Spin Column Total RNA Mini-Preps Super Kit (Bio Basic Inc., Amherst, NY, USA). RT-PCR was performed on an Applied Biosystems Two-Step Real-Time PCR System (Thermo Fisher Scientific, Inc.) using DNAse I (Thermo Fisher Scientific, Inc.) using the SYBR Green Realtime PCR Master Mix (Toyobo, Osaka, Japan). PCR was performed under the following conditions: $95^{\circ} \mathrm{C}$ for $10 \mathrm{~min}$, followed by 40 cycles of $95^{\circ} \mathrm{C}$ for $15 \mathrm{sec}$ and $58^{\circ} \mathrm{C}$ for $60 \mathrm{sec}$. The primer sequences were as follows: Mfn2 forward, 5'-GCT CGGAGGCACATGAAAGT-3' and reverse, 5'-ATCACGGTG CTCTTCCCATT-3'; and $\beta$-actin forward, 5'-GACAGTGTT GTGGGTGTAGGTACTAAC-3' and reverse, 5'-CCGCTTTAC ACCAGCGTCAT-3'. $\beta$-actin was used as the internal control. Relative mRNA expression levels of Mfn2 were determined using the $2^{-\Delta \Delta \mathrm{Cq}}$ method, as described previously (19).

Electron microscopy. The mitochondria in $\mathrm{C} 2 \mathrm{C} 12$ cells were observed by electron microscopy using conventional fixation-embedding procedures (20).

Hematoxylin-eosin (HE) staining. The gastrocnemius muscles from the mice were embedded in paraffin and cut into $6-\mu \mathrm{m}$ tissue sections, which were then stained with HE (Beyotime Institute of Biotechnology), according to the manufacturer's protocol. Subsequently, the tissue sections were observed under a light microscope.

Statistical analysis. Statistical analyses involved the unpaired two-tailed Student's t-test, two-way analysis of variance and the Student-Newman-Keuls method, which were performed using GraphPad Prism 5.0 software (GraphPad Software, Inc., La Jolla, CA, USA). Data are presented as the mean \pm standard error of the mean of three independent experiments. $\mathrm{P}<0.05$ was considered to indicate a statistically significant difference.

\section{Results}

Downregulation of Mfn2 in the rectus abdominis is associated with body weight loss in patients with gastrointestinal cancer. The present study assessed the association between Mfn2 levels in the rectus abdominis and the body weight loss in 21 patients with gastric or colorectal cancer. Fig. 1 shows the percentage body weight loss in the 21 patients at 6 months prior to surgery. The patients were numbered according to their weight loss. Patient 1 lost $20 \%$ body weight, while patients 19 , 20 and 21 showed little weight loss (Fig. 1A). The expression levels of Mfn2 in the rectus abdominis of the 21 patients were determined by western blotting (Fig. 1B). The patients were divided into two groups: Patients (nos. 1-13) who showed $>5 \%$ weight loss were in the cachexia period and those who showed $<5 \%$ weight loss were in the pre-cachexia period. The relative protein expression levels of Mfn2 in the patients with cachexia were significantly lower compared with the pre-cachexia patients $(\mathrm{P}<0.001$; Fig. 1C). In addition, the downregulation of Mfn2 expression in the rectus abdominis of patients with cachexia was associated with weight loss (Fig. 1D). These results suggest that Mfn2 is involved in cachexia and its downregulation is associated with the progression of cachexia. 
A

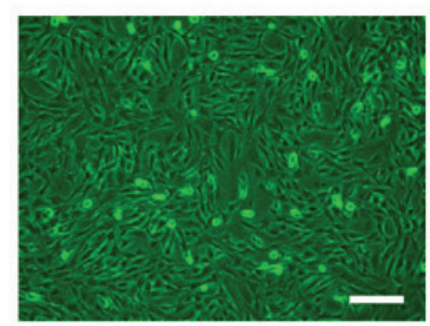

E

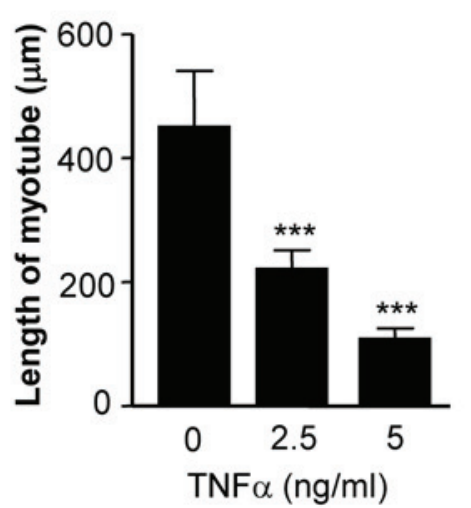

B

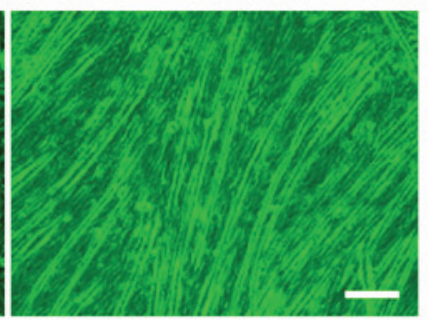

C

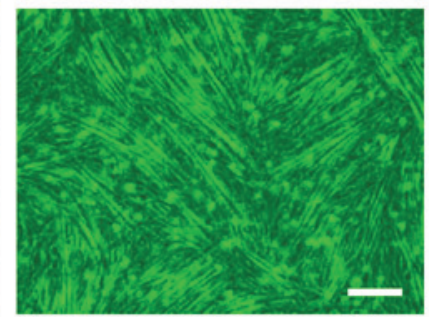

D

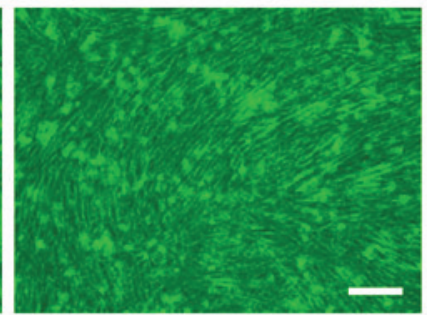

F

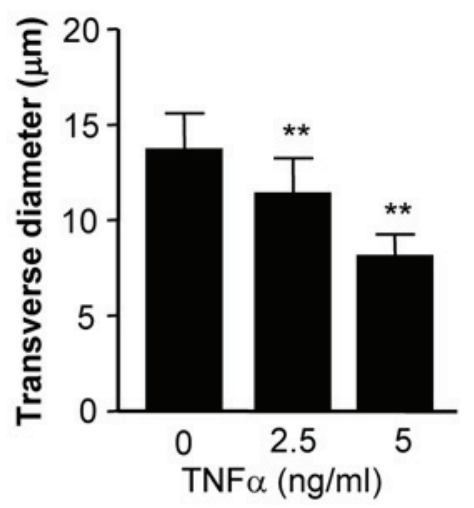

G

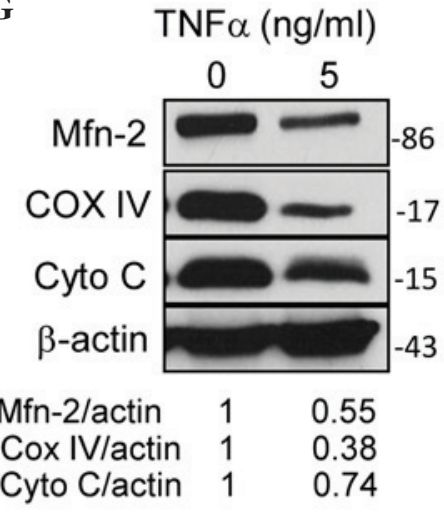

Figure 2. Expression of Mfn2 was downregulated following TNF- $\alpha$-induced myotube atrophy of C2C12 cells. (A) Undifferentiated C2C12 cells, (B) C2C12 cells differentiated for 7 days, (C) C2C12 cells differentiated for 7 days in the presence of $2.5 \mathrm{ng} / \mathrm{ml} \mathrm{TNF}-\alpha$ and (D) C2C12 cells differentiated for 7 days in the presence of $5 \mathrm{ng} / \mathrm{ml} \mathrm{TNF}-\alpha$. (E) Length of myotube formed by differentiated $\mathrm{C} 2 \mathrm{C} 12$ cells. (F) Transverse diameter of myotube formed by differentiated $\mathrm{C} 2 \mathrm{C} 12$ cells. (G) Protein expression levels of Mfn2, COX IV, Cyto C and $\beta$-actin in $\mathrm{C} 2 \mathrm{C} 12$ cells and differentiated C2C12 cells treated with or without TNF- $\alpha$. ${ }^{* *} \mathrm{P}<0.01,{ }^{* * *} \mathrm{P}<0.001$ vs. the control ( $\left.0 \mathrm{ng} / \mathrm{ml} \mathrm{TNF}-\alpha\right)$. Mfn2, mitofusin-2; TNF- $\alpha$, tumor necrosis factor- $\alpha$; COX-IV, cytochrome $c$ oxidase subunit IV; Cyto C, cytochrome $c$.

Expression of Mfn2 is downregulated in TNF- $\alpha$-induced myotube atrophy of C2C12 cells. C2C12 cells are an immortalized cell line of mouse skeletal muscle cells that fuse and differentiate into myotube under low-serum conditions (21). TNF- $\alpha$ has a critical role in muscle loss during cancer cachexia (22), and TNF- $\alpha$-treated differentiated C2C12 cells have previously been used as an in vitro model of muscle atrophy (23). The present study used this model to assess the expression of $\mathrm{Mfn} 2$ in differentiated $\mathrm{C} 2 \mathrm{C} 12$ cells treated with or without TNF- $\alpha$. Fig. 2A shows $\mathrm{C} 2 \mathrm{C} 12$ cells prior to differentation and Fig. 2B shows the differentiated cells with normal myotube formation. In a previous study, TNF- $\alpha$ was able to reduce the expression of Mfn2 in cultured cells (16). In the present study, the addition of TNF- $\alpha$ prevented the formation of normal myotube (Fig. 2C and D). Specifically, TNF- $\alpha$ treatment reduced the length (Fig. 2E) and transverse diameter (Fig. 2F) of myotube, suggesting that TNF- $\alpha$ induces myotube atrophy. Furthermore, the expression levels of Mfn2 in TNF- $\alpha$-treated C2C12 cells were examined, and it was demonstrated that TNF- $\alpha$ inhibited the expression of Mfn2 (Fig. 2G).

The mitochondrial proteins COX IV and Cyto $\mathrm{C}$ are two key proteins of the mitochondrial respiratory chain, which has a critical role in mitochondrial function (11). The expression levels of COX IV and Cyto $\mathrm{C}$ have been used to assess the mitochondrial function of cells in previous studies $(11,24)$. Therefore, the present study determined the expression levels of COX IV and Cyto C in C2C12 cells, and demonstrated that the expression of these two proteins were decreased (Fig. 2H), indicating that the mitochondrial function of the cells was impaired.

Overexpression of Mfn2 ameliorates TNF-induced myotube atrophy of $\mathrm{C} 2 \mathrm{C} 12$ cells. To assess whether Mfn2 is required for the TNF- $\alpha$-induced myotube atrophy of $\mathrm{C} 2 \mathrm{C} 12$ cells, Mfn2 was overexpressed in $\mathrm{C} 2 \mathrm{C} 12$ cells. It was observed that overexpression of Mfn2 prevented the TNF- $\alpha$-mediated induction of myotube atrophy (Fig. 3A and B). In addition, overexpression of Mfn2 upregulated the expression of COX IV and Cyto C in $\mathrm{C} 2 \mathrm{C} 12$ cells (Fig. 3C), suggesting the recovery of mitochondrial function. Furthermore, the appearance of the mitochondria in $\mathrm{C} 2 \mathrm{C} 12$ cells prior to and following TNF- $\alpha$ treatment was examined. Prior to treatment, $\mathrm{C} 2 \mathrm{C} 12$ cells had mitochondria of a normal size with cristae (Fig. 4A), whereas TNF- $\alpha$ treatment led to abnormal and fragmented mitochondria in cells (Fig. 4B). Overexpression of $\mathrm{Mfn} 2$ in $\mathrm{C} 2 \mathrm{C} 12$ cells partially attenuated the damage to mitochondria (Fig. 4C). In addition, the lengths of mitochondria were measured (Fig. 4D), and TNF- $\alpha$ was shown to decrease the average length of mitochondria in $\mathrm{C} 2 \mathrm{C} 12$ cells, which was reversed in part by overexpression of Mfn2.

Overexpression of $M f n 2$ prevents cachexia-induced gastrocnemius loss in mice. The potential role of Mfn2 in cachexia was assessed using a nude mouse model. Mice were injected subcutaneously with HCT116 cells to promote the growth of tumor xenografts. Notably, xenograft growth was associated with weight loss in the mice (Fig. 5A). Mice were categorized as having severe cachexia if the body weights were $>2$ SDs 


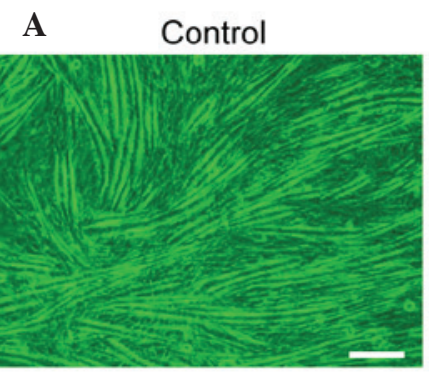

$\mathbf{E}$

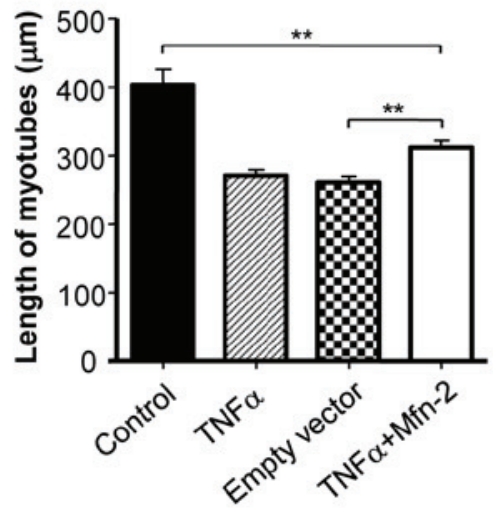

$\mathrm{TNF} \alpha$

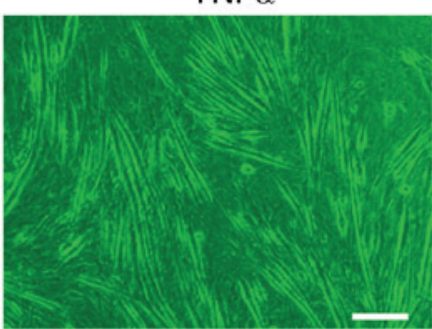

$\mathbf{F}$

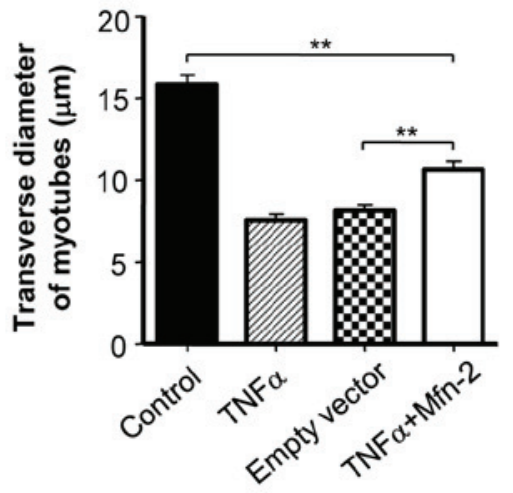

C Empty vector

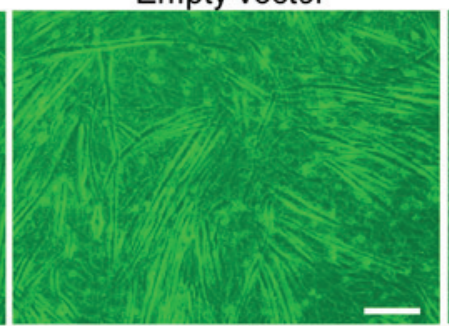

G

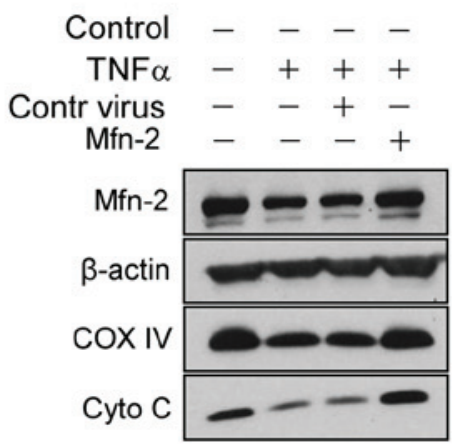

Figure 3. Overexpression of Mfn2 ameliorates TNF- $\alpha$-induced myotube atrophy of $\mathrm{C} 2 \mathrm{C} 12$ cells. (A) $\mathrm{C} 2 \mathrm{C} 12$ cells were cultured in differentiation medium for 7 days. (B) $\mathrm{C} 2 \mathrm{C} 12$ cells were cultured in differentiation medium for 7 days in the presence of $5 \mathrm{ng} / \mathrm{ml}$ TNF- $\alpha$. (C) $\mathrm{C} 2 \mathrm{C} 12$ cells were infected with control lentivirus and then cultured with differentiation medium for 7 days in the presence of $5 \mathrm{ng} / \mathrm{ml} \mathrm{TNF}-\alpha$. (D) C2C12 cells were infected with Mfn2 lentivirus and then cultured in differentiation medium in the presence of $5 \mathrm{ng} / \mathrm{ml}$ TNF- $\alpha$ for 7 days. (E) Determination of C2C12 myotube length. (F) Determination of myotube transverse diameter. (G) The expression levels of Mfn2, COX IV, Cyto C and $\beta$-actin in C2C12 cells that received various treatments were determined using western blotting. Data are expressed as the mean \pm standard error of the mean. ${ }^{* *} \mathrm{P}<0.01$. Mfn2, mitofusin-2; TNF- $\alpha$, tumor necrosis factor- $\alpha$; COX-IV, cytochrome $c$ oxidase subunit IV; Cyto C, cytochrome $c$.

A

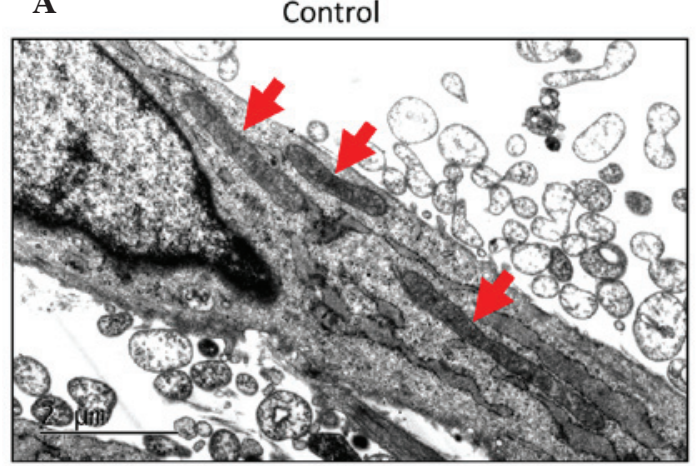

C

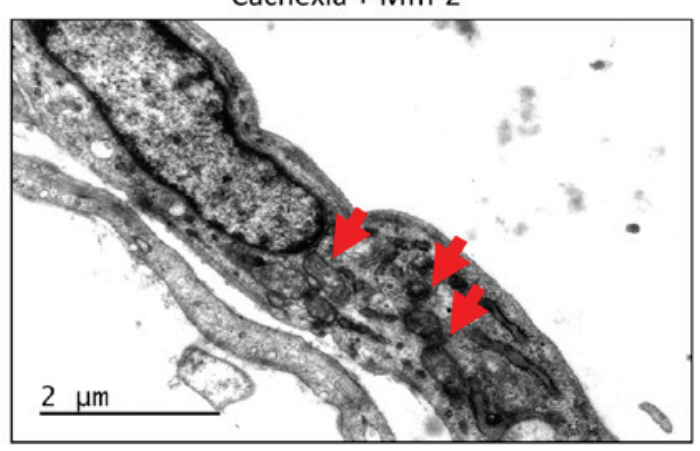

B

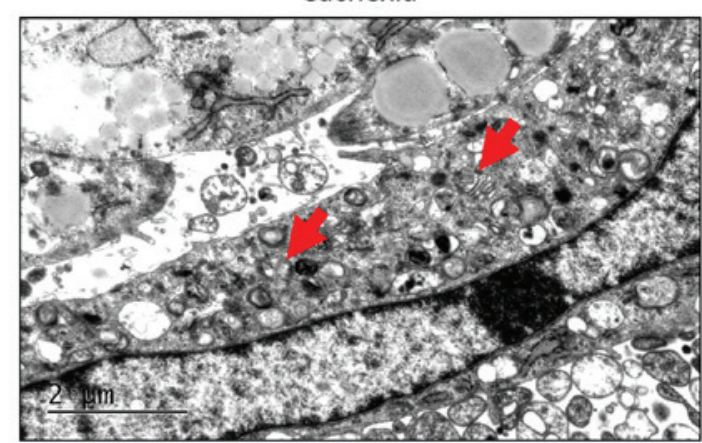

D

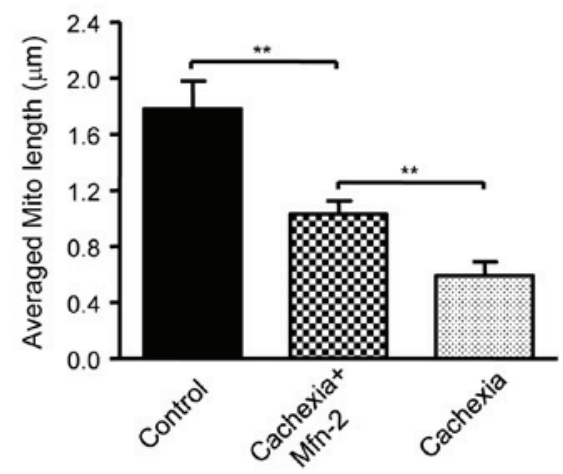

Figure 4. Expression of Mfn2 inhibited TNF- $\alpha$-induced fragmentation of mitochondria. (A) Electron microscopy of C2C12 cells cultured in differentiation medium for 7 days. (B) $\mathrm{C} 2 \mathrm{C} 12$ cells were differentiated for 7 days in the presence of $5 \mathrm{ng} / \mathrm{ml} \mathrm{TNF}-\alpha$. (C) $\mathrm{C} 2 \mathrm{C} 12$ cells were infected with Mfn2 lentivirus, and then cultured in differentiation medium containing $5 \mathrm{ng} / \mathrm{ml} \mathrm{TNF}-\alpha$ days. The red arrows indicate mitochondria in cells. (D) Measurement of the length of mitochondria in $\mathrm{C} 2 \mathrm{C} 12$ cells. ${ }^{* * *} \mathrm{P}<0.01$. Mfn2, mitofusin-2; TNF- $\alpha$, tumor necrosis factor- $\alpha$. 
A

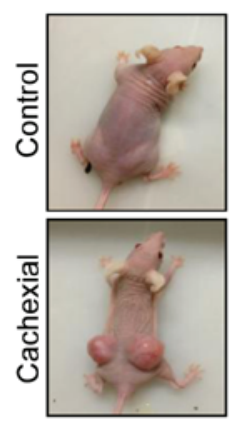

B

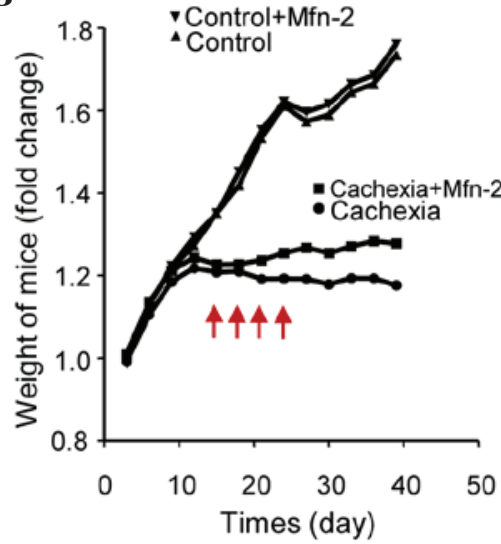

C

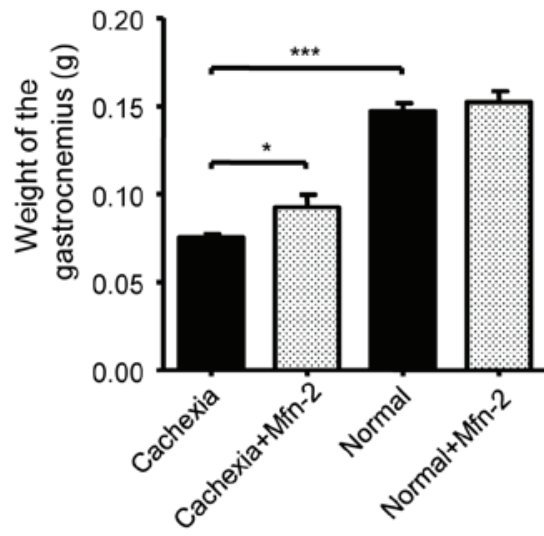

D

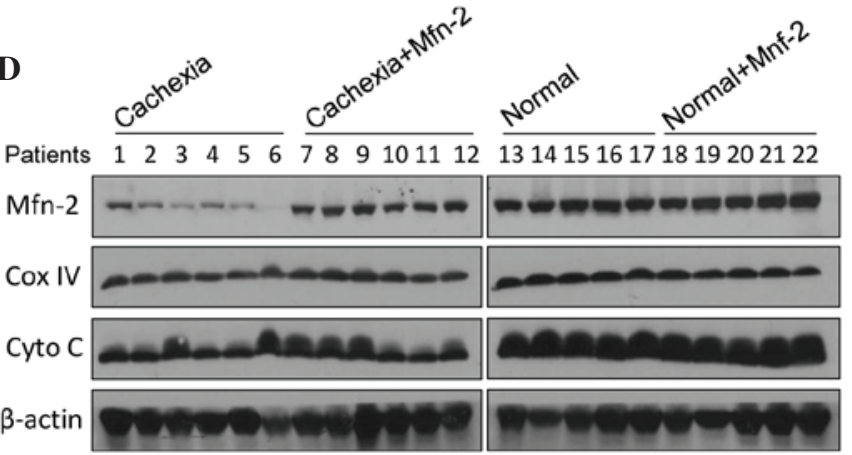

$\mathbf{E}$

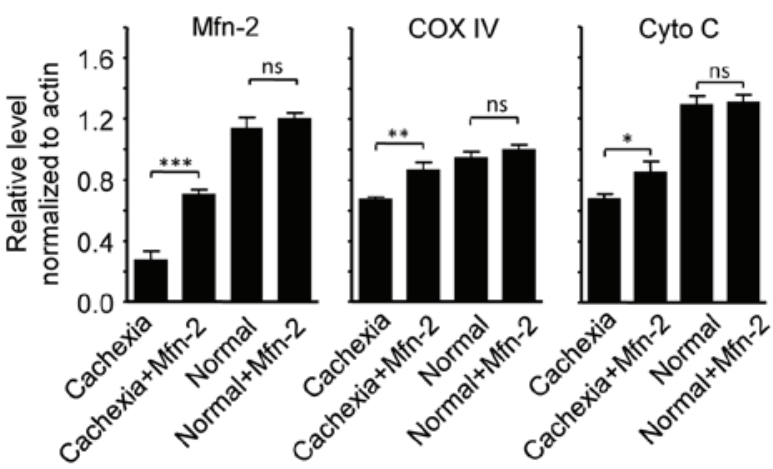

Figure 5. Overexpression of Mfn2 prevents cachexia-induced gastrocnemius muscle loss in a mouse model. (A) Representative images of the control and cachexia nude mice. (B) Body weights of the nude mice. The red arrows indicate the time at which the Mfn2 lentivirus was injected into the gastrocnemius muscles of the Mfn2-treated mice. (C) After 40 days, the mice were sacrificed and the gastrocnemius muscles of the mice were excised and weighed. (D) The protein expression of Mfn2, COX IV, Cyto $\mathrm{C}$ and $\beta$-actin in the mice gastrocnemius was evaluated by western blotting. (E) The relative expression levels of Mfn2, COX IV and Cyto $\mathrm{C}$ in the mice gastrocnemius were determined by densitometry. ${ }^{*} \mathrm{P}<0.05,{ }^{* *} \mathrm{P}<0.01,{ }^{* * *} \mathrm{P}<0.001$ vs. the cachexia mice. Mfn2, mitofusin-2; COX-IV, cytochrome $c$ oxidase subunit IV; Cyto C, cytochrome $c$.

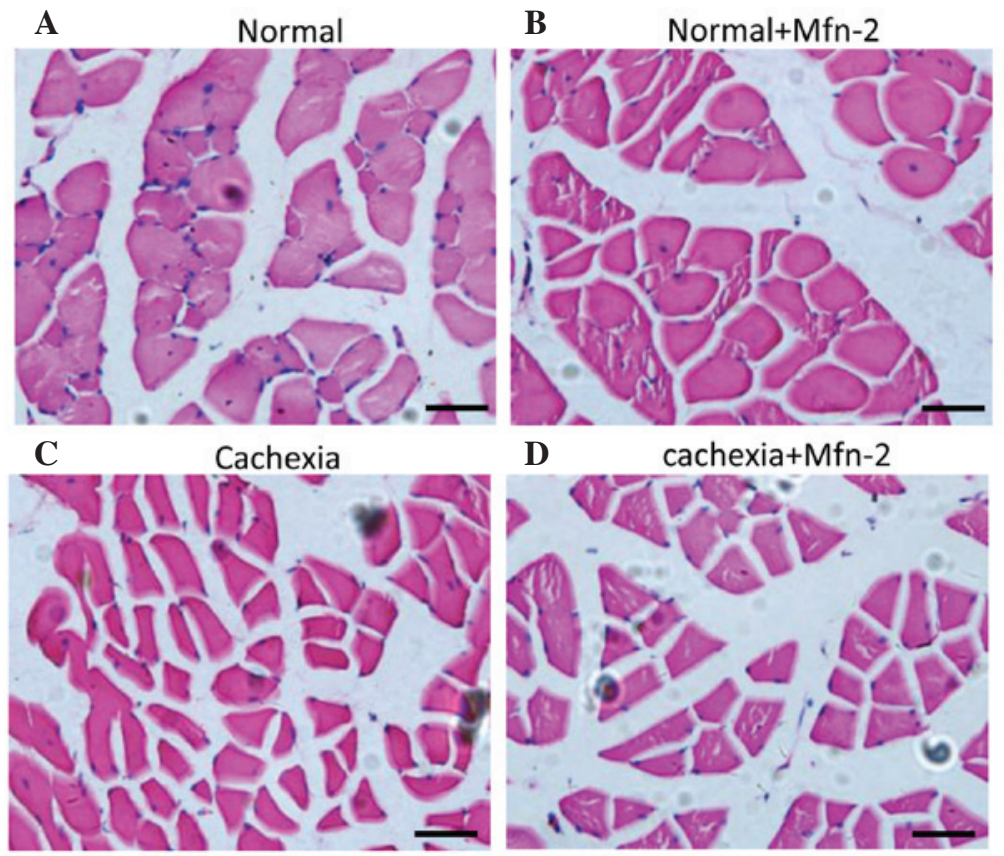

$\mathbf{E}$

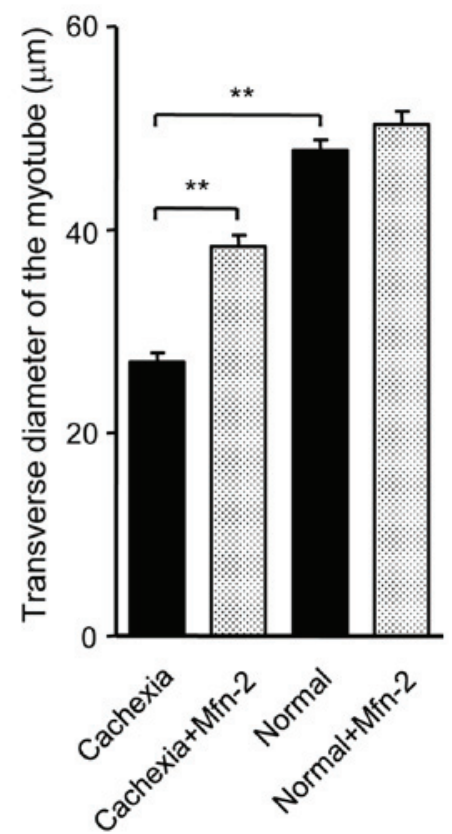

Figure 6. Overexpression of Mfn2 inhibits cachexia. (A) Hematoxylin \& eosin (HE) staining of the gastrocnemius from control mice injected with empty lentivirus. (B) HE staining of the gastrocnemius from control mice injected with the Mfn2 lentivirus. (C) HE staining of the gastrocnemius from cachexia mice injected with control lentivirus. (D) HE staining of the gastrocnemius from cachexia mice injected with Mfn2 lentivirus. (E) Measurement of transverse diameter of myotube of gastrocnemius muscles. ${ }^{* *} \mathrm{P}<0.01$ vs. the cachexia mice. Mfn2, mitofusin- 2 . 
away from the means of age-matched control mice. Empty or Mfn2 lentivirus were injected into the gastrocnemius of these mice every 3 days, four times in total (Fig. 5A). After 40 days, the mice were sacrificed and the gastrocnemius muscles were excised and weighed. The protein expression levels of Mfn2 in the mouse gastrocnemius muscles are shown in Fig. 5B. It was observed that the weight of the gastrocnemius muscle in mice with cachexia was significantly decreased, as compared with the control mice $(\mathrm{P}<0.001$; Fig. $5 \mathrm{C})$. Notably, treatment of the cachexia mice with Mfn2 lentivirus significantly increased the weight of the gastrocnemius muscle, as compared with the untreated cachexia mice $(\mathrm{P}<0.05$; Fig. $5 \mathrm{C})$. HE staining of gastrocnemius muscle tissue sections indicated that the morphology of the control and control + Mfn2 gastrocnemius muscle was normal (Fig. 6A and B). Conversely, staining of the gastrocnemius muscle from mice with cachexia showed obvious atrophy of the muscle (Fig. 6C). Overexpression of Mfn2 partially ameliorated the cachexia-associated morphological changes of the muscle (Fig. 6D). The transverse diameters of the myotube were measured and are shown in Fig. 6E. It was observed that the transverse diameters of the myotube of the gastrocnemius muscles in mice with cachexia were significantly decreased when compared with the control mice $(\mathrm{P}<0.01$; Fig. 6E). Notably, treatment of the cachexia mice with Mfn2 lentivirus significantly increased the transverse diameters of the myotube compared with the untreated cachexia mice $(\mathrm{P}<0.01$; Fig. $6 \mathrm{E})$.

\section{Discussion}

Cancer cachexia remains a leading cause of morbidity and mortality worldwide, despite extensive research and clinical trials, and is identified in $\leq 80 \%$ of upper gastrointestinal cancer patients and $60 \%$ of lung cancer patients at the time of diagnosis (5). The prominent clinical feature of cancer cachexia is the continuous loss of skeletal muscle that cannot be fully reversed by conventional nutritional support, and which leads to progressive functional impairment (2). However, the underlying mechanism remains largely unknown. The present study demonstrated that Mfn2 was downregulated in the gastrocnemius muscles of patients with gastrointestinal cancer, which was associated with weight loss. In vitro cell tests and in vivo animal experiments suggested that overexpression of Mfn2 was able to reverse cachexia-induced muscle wasting. These results suggested that Mfn2 has an important role in skeletal muscle loss in patients with cancer cachexia, and that Mfn2 may be a novel target for prevention of skeletal muscle loss caused by cachexia.

Loss of skeletal muscle is the major symptom of cancer cachexia, which leads to reduced mobility, a low quality of life and a decreased life expectancy (5). The exact mechanism by which cancer cachexia causes skeletal muscle loss is poorly understood. A previous study suggested that inflammatory cytokines such as TNF- $\alpha$, interferon (IFN)- $\gamma$ and interleukin (IL)-6 likely have a role in muscle loss during cancer cachexia (25). In addition, dysfunction of the mitochondria of skeletal muscle may also explain the muscle loss (26). Mfn2 has an important role in the regulation of mitochondrial function $(14,15)$. Therefore, we hypothesized that Mfn2 may have a role in muscle wasting caused by cachexia. In order to investigate this, the expression levels of Mfn2 in the rectus abdominis of cancer patients with cachexia were determined. It was shown that Mfn2 was downregulated in the rectus abdominis, which was associated with the severity of muscle loss in these patients. These results suggested that downregulation of Mfn2 expression is associated with the progression of muscle wasting in patients with cancer cachexia. The in vitro cell experiments showed that a reduction in Mfn2 expression was observed in TNF- $\alpha$-induced $\mathrm{C} 2 \mathrm{C} 12$ myotube atrophy, and in vivo animal tests indicated that loss of Mfn2 was associated with gastrocnemius muscle mass loss in tumor-bearing mice. Notably, it was demonstrated that overexpression of Mfn2 in $\mathrm{C} 2 \mathrm{C} 12$ cells was able to reverse TNF- $\alpha$-induced myotube atrophy, and re-introduction of this gene into mice gastrocnemius could reverse cachexia-induced muscle wasting. Together, these results suggested that Mfn2 has a critical role in skeletal muscle wasting in cancer cachexia.

The results of the present study suggested that Mfn2 has a crucial role in muscle wasting during cachexia; however, the underlying mechanism was unclear. The loss of fusion proteins may predispose the mitochondria to undergo fragmentation (27). Mfn2 participates in mitochondrial fusion and contributes to the maintenance and operation of the mitochondrial network; thus it is possible that a decrease in the expression of Mfn2 in muscle cells may lead to mitochondrial dysfunction and thereby muscle wasting. In the present study, a reduction in the expression levels of COX IV and Cyto $\mathrm{C}$ was observed concomitantly with the downregulation of Mfn2. COX IV and Cyto C are proteins of the mitochondrial respiratory chain and their loss results in reduced ATP formation and mitochondrial dysfunction (11). Therefore, it is possible that cancer cachexia induces the downregulation of Mfn2 in skeletal muscle cells, leading to mitochondrial dysfunction and thereafter muscle mass loss.

Previous studies have reported that inflammatory cytokines, such as TNF- $\alpha$, IFN $\gamma$ and IL-6, likely have a role in the occurrence of muscle wasting in patients with cancer cachexia $(11,22,23,25)$. In addition, increased levels of inflammatory cytokines have been observed in patients with cancer cachexia $(7,28)$. Therefore, it may be hypothesized that inflammatory factors are involved in the downregulation of Mfn2 expression in cancer cachexia. In the present study, treatment of $\mathrm{C} 2 \mathrm{C} 12$ cells with $\mathrm{TNF}-\alpha$ resulted in the downregulation of $\mathrm{Mfn} 2$ protein expression levels. Additional studies are required to determine the underlying molecular mechanisms.

In conclusion, the present study demonstrated that downregulation of Mfn2 was associated with skeletal muscle loss in cancer cachexia, and that overexpression of Mfn2 was able to ameliorate this loss. These results suggested that Mfn2 protects against cachexia-induced muscle loss, and that Mfn2 may be a novel target for the treatment of cachexia in cancer patients.

\section{Acknowledgements}

The present study was supported by the National Natural Science Foundation of China (grant no. 81372197). 


\section{References}

1. Fearon KC, Voss AC and Hustead DS; Cancer Cachexia Study Group: Definition of cancer cachexia: Effect of weight loss, reduced food intake, and systemic inflammation on functional status and prognosis. Am J Clin Nutr 83: 1345-1350, 2006.

2. Fearon K, Strasser F, Anker SD, Bosaeus I, Bruera E, Fainsinger RL, Jatoi A, Loprinzi C, MacDonald N, Mantovani G, et al: Definition and classification of cancer cachexia: An international consensus. Lancet Oncol 12: 489-495, 2011

3. Bachmann J, Heiligensetzer M, Krakowski-Roosen $\mathrm{H}$, Büchler MW, Friess H and Martignoni ME: Cachexia worsens prognosis in patients with resectable pancreatic cancer. J Gastrointest Surg 12: 1193-1201, 2008.

4. Dewys WD, Begg C, Lavin PT, Band PR, Bennett JM, Bertino JR, Cohen MH, Douglass HO Jr, Engstrom PF, Ezdinli EZ, et al: Prognostic effect of weight loss prior to chemotherapy in cancer patients. Eastern Cooperative Oncology Group. Am J Med 69: 491-497, 1980.

5. Donohoe CL, Ryan AM and Reynolds JV: Cancer cachexia: Mechanisms and clinical implications. Gastroenterol Res Pract 2011: 601434, 2011.

6. Bozzetti F, Arends J, Lundholm K, Micklewright A, Zurcher G and Muscaritoli M; ESPEN: ESPEN guidelines on parenteral nutrition: Non-surgical oncology. Clin Nutr 28: 445-454, 2009.

7. Deans DA, Tan BH, Wigmore SJ, Ross JA, de Beaux AC, Paterson-Brown S and Fearon KC: The influence of systemic inflammation, dietary intake and stage of disease on rate of weight loss in patients with gastro-oesophageal cancer. $\mathrm{Br} J$ Cancer 100: 63-69, 2009.

8. Romanello V, Guadagnin E, Gomes L, Roder I, Sandri C, Petersen Y, Milan G, Masiero E, Del Piccolo P, Foretz M, et al: Mitochondrial fission and remodelling contributes to muscle atrophy. EMBO J 29: 1774-1785, 2010.

9. Soriano FX, Liesa M, Bach D, Chan DC, Palacín M and Zorzano A: Evidence for a mitochondrial regulatory pathway defined by peroxisome proliferator-activated receptor-gamma coactivator-1alpha, estrogen-related receptor-alpha, and mitofusin 2. Diabetes 55: 1783-1791, 2006.

10. Huang P, Galloway CA and Yoon Y: Control of mitochondrial morphology through differential interactions of mitochondrial fusion and fission proteins. PLoS One 6: e20655, 2011.

11. White JP, Baltgalvis KA, Puppa MJ, Sato S, Baynes JW and Carson JA: Muscle oxidative capacity during IL-6-dependent cancer cachexia. Am J Physiol Regul Integr Comp Physiol 300: R201-R211, 2011.

12. Shen T, Zheng M, Cao C, Chen C, Tang J, Zhang W, Cheng H, Chen KH and Xiao RP: Mitofusin-2 is a major determinant of oxidative stress-mediated heart muscle cell apoptosis. J Biol Chem 282: 23354-23361, 2007.

13. Song Z, Ghochani M, McCaffery JM, Frey TG and Chan DC: Mitofusins and OPA1 mediate sequential steps in mitochondrial membrane fusion. Mol Biol Cell 20: 3525-3532, 2009.
14. Ranieri M, Brajkovic S, Riboldi G, Ronchi D, Rizzo F, Bresolin N, Corti S and Comi GP: Mitochondrial fusion proteins and human diseases. Neurol Res Int 2013: 293893, 2013.

15. Kluge MA, Fetterman JL and Vita JA: Mitochondria and endothelial function. Circ Res 112: 1171-1188, 2013.

16. Bach D, Naon D, Pich S, Soriano FX, Vega N, Rieusset J, Laville M, Guillet C, Boirie Y, Wallberg-Henriksson H, et al: Expression of Mfn2, the Charcot-Marie-Tooth neuropathy type 2A gene, in human skeletal muscle: Effects of type 2 diabetes, obesity, weight loss, and the regulatory role of tumor necrosis factor alpha and interleukin-6. Diabetes 54: 2685-2693, 2005.

17. Hernández-Alvarez MI, Thabit H, Burns N, Shah S, Brema I, Hatunic M, Finucane F, Liesa M, Chiellini C, Naon D, et al: Subjects with early-onset type 2 diabetes show defective activation of the skeletal muscle PGC-1\{alpha\}/Mitofusin-2 regulatory pathway in response to physical activity. Diabetes Care 33: 645-651, 2010

18. Zhang HS, Chen Y, Fan L1, Xi QL, Wu GH, Li XX, Yuan TL, He SQ, Yu Y1, Shao ML, Liu Y, et al: The endoplasmic reticulum stress sensor IRE1 $\alpha$ in intestinal epithelial cells is essential for protecting against colitis. J Biol Chem 290: 15327-15336, 2015.

19. Xue J, Li X, Jiao S, Wei Y, Wu G and Fang J: Prolyl hydroxylase-3 is down-regulated in colorectal cancer cells and inhibits IKKbeta independent of hydroxylase activity. Gastroenterology 138: 606-615, 2010.

20. Eura Y, Ishihara N, Yokota S and Mihara K: Two mitofusin proteins, mammalian homologues of $\mathrm{FZO}$, with distinct functions are both required for mitochondrial fusion. J Biochem 134: 333-344, 2003.

21. Yaffe D and Saxel O: Serial passaging and differentiation of myogenic cells isolated from dystrophic mouse muscle. Nature 270: 725-727, 1977.

22. Reid MB and Li YP: Tumor necrosis factor-alpha and muscle wasting: A cellular perspective. Respir Res 2: 269-272, 2001.

23. De Larichaudy J, Zufferli A, Serra F, Isidori AM, Naro F, Dessalle K, Desgeorges M, Piraud M, Cheillan D, Vidal H, et al: TNF- $\alpha$ - and tumor-induced skeletal muscle atrophy involves sphingolipid metabolism. Skelet Muscle 2: 2, 2012.

24. Wu Z, Puigserver P, Andersson U, Zhang C, Adelmant G, Mootha V, Troy A, Cinti S, Lowell B, Scarpulla RC, Spiegelman BM: Mechanisms controlling mitochondrial biogenesis and respiration through the thermogenic coactivator PGC-1. Cell 98: 115-124, 1999.

25. Tisdale MJ: Wasting in cancer. J Nutr 129 (1S Suppl): S243-S246, 1999.

26. Argilés JM, Busquets S, Stemmler B and López-Soriano FJ: Cancer cachexia: Understanding the molecular basis. Nat Rev Cancer 14: 754-762, 2014.

27. Sugioka R, Shimizu S and Tsujimoto Y: Fzo1, A protein involved in mitochondrial fusion, inhibits apoptosis. J Biol Chem 279: 52726-52734, 2004

28. Deans C and Wigmore SJ: Systemic inflammation, cachexia and prognosis in patients with cancer. Curr Opin Clin Nutr Metab Care 8: 265-269, 2005. 